\title{
Coherent Plasmon-Exciton Coupling in Silver Platelet-J-aggregate Nanocomposites
}

\author{
Brendan G. DeLacy, ${ }^{*}{ }^{\dagger}$ Owen D. Miller, ${ }^{\ddagger}$ Chia Wei Hsu, ${ }^{\S}, \|$ Zachary Zander, ${ }^{\dagger}$ Steven Lacey, ${ }^{\dagger}$ \\ Raymond Yagloski, ${ }^{\dagger}$ Augustus W. Fountain, ${ }^{\dagger}$ Erica Valdes, ${ }^{\dagger}$ Emma Anquillare, ${ }^{\S}$ Marin Soljačić, ${ }^{\S}$ \\ Steven G. Johnson, ${ }^{\ddagger}$ and John D. Joannopoulos ${ }^{\S}$
}

${ }^{\dagger}$ U.S. Army Edgewood Chemical Biological Center, Aberdeen Proving Ground, Maryland 21010, United States

${ }^{\ddagger}$ Department of Mathematics and ${ }^{\S}$ Department of Physics, Massachusetts Institute of Technology, Cambridge, Massachusetts 02139, United States

"Department of Physics, Harvard University, Cambridge, Massachusetts 02138, United States

ABSTRACT: Hybrid nanostructures that couple plasmon and exciton resonances generate hybridized energy states, called plexcitons, which may result in unusual light-matter interactions. We report the formation of a transparency dip in the visible spectra of colloidal suspensions containing silver nanoplatelets and a cyanine dye, 1,1'-diethyl-2,2' -cyanine iodide (PIC). PIC was electrostatically adsorbed onto the surface of silver nanoplatelet core particles, forming an outer J-aggregate shell. This core-shell architecture provided a framework for coupling the plasmon resonance of the silver nanoplatelet core with the exciton resonance of the J-aggregate shell. The sizes and aspect ratios of the silver nanoplatelets were controlled to ensure the overlap of the plasmon and exciton resonances. As a measure of the plasmon-exciton coupling strength in the system, the experimentally observed transparency dips correspond to a Rabi splitting energy of $207 \mathrm{meV}$, among the highest reported for colloidal nanoparticles. The optical properties of the silver platelet-J-aggregate nanocomposites were

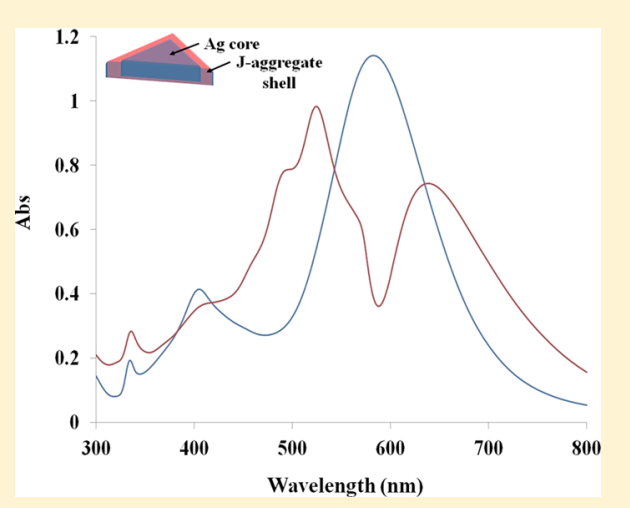
supported numerically and analytically by the boundary-element method and temporal coupled-mode theory, respectively. Our theoretical predictions and experimental results confirm the presence of a transparency dip for the silver nanoplatelet core Jaggregate shell structures. Additionally, the numerical and analytical calculations indicate that the observed transparencies are dominated by the coupling of absorptive resonances, as opposed to the coupling of scattering resonances. Hence, we describe the suppressed extinction in this study as an induced transparency rather than a Fano resonance.

KEYWORDS: Plexcitons, plasmons, excitons, J-aggregates

$\mathrm{P}$ lasmon-exciton coupling in multilayered nanostructures has garnered much attention in recent years, due to the tunable and unique optical properties that these structures exhibit. These hybrid systems often consist of a core-shell geometry in which the localized surface plasmon resonance (LSPR) of the metallic core couples with the exciton resonance exhibited by a J-aggregate dye or a quantum dot shell. ${ }^{1-10}$ This architecture provides a means of studying plasmon-exciton interactions, which have resulted in unique optical phenomena such as induced transparency. ${ }^{1,2,5,6,11,12}$ The ability to control the morphology and dimensions of the individual layers at the nanoscale and the subsequent control of optical properties are ultimately what drive this field of research. Plexcitonic research, although a relatively new field, has resulted in its use in chemical sensors, light harvesting devices, and optical devices. $^{13-16}$

Metal/cyanine dye hybrid nanostructures are particularly suitable for studying plasmon-exciton interactions in core-shell geometries due to the relative ease with which the plasmonic nanoparticle morphology and size may be tuned to ensure the overlap of plasmon and exciton resonances. Significant progress in the fabrication and control over the morphology of plasmonic gold and silver nanoparticles has been made in recent years. These efforts have resulted in reliable methods for the fabrication of plasmonic nanorods, nanocubes, nanostars, and nanoplatelets. ${ }^{17-20}$

Cyanine dyes are another class of materials that have been extensively studied due to their use in spectral sensitization and potential applications in novel optoelectronic materials. These dyes have a tendency to aggregate under reduced solubility conditions or when adsorption occurs on particle or substrate surfaces. The J-aggregates that are formed exhibit a narrow line shape that is red-shifted relative to the monomer absorption band. A Frenkel exciton model is often used to describe this shift as a result of excited states that are formed by the coherent coupling of molecular transition dipoles. ${ }^{21,22}$ The tendency of

Received: January 14, 2015

Revised: February 26, 2015

Published: February 27, 2015 
cyanine dyes to aggregate on the surface of metallic nanoparticles makes them ideal candidates as excitonic shells on plasmonic core nanoparticles.

In this Letter, we explore plasmon-exciton coupling in colloidal suspensions containing silver nanoplatelet-J-aggregate nanocomposites with core-shell structures. The nanostructures were fabricated by electrostatically adsorbing $1,1^{\prime}$-diethyl-2,2'cyanine iodide (PIC) onto the surface of silver nanoplatelets. The silver nanoplatelet core geometry was chosen for its optical properties and dimensions, which were tuned to ensure the overlap of the plasmonic resonance with the excitonic resonance of the PIC J-aggregate. The overall goal of this study was to ascertain whether or not an induced transparency could be generated using a plasmonic nanoplatelet-J-aggregate core-shell geometry.

Induced transparency is a term that is collectively used to describe the suppressed extinction that is exhibited by a multiresonant structure. In the uncoupled state, the individual resonances yield the extinction of light for a given band of incident radiation wavelengths. Conversely, when multiple plasmonic resonances, plasmonic and excitonic resonances, or dielectric resonances overlap and are coupled, an induced transparency may be observed. A plethora of approaches have been taken to model this transparency phenomenon, including perturbative models, ${ }^{23}$ a generalization of the Fano formula, ${ }^{24-26}$ the electrostatic approximation, ${ }^{27,28}$ and coupledmechanical-oscillator models. ${ }^{29-33}$ For those multiresonant structures that are scattering dominant, a Fano resonance is often used to describe the induced transparency line shape that is the result of the destructive interference of scattered and transmitted light. ${ }^{34}$ Recently, an analytical treatment of light scattering from a multiresonant nanostructure was developed using temporal-coupled mode theory. ${ }^{35}$ In this study, we use both the boundary-element method and temporal-coupled mode theory to describe the plasmon-exciton coupling observed in the silver platelet/J-aggregate nanocomposite. Specifically, we seek to use these approaches to determine if the induced transparency observed in our silver platelet/Jaggregate system is dominated by the coupling of scattering resonances, that is, a Fano resonance, or if the induced transparency is dominated by the coupling of two absorption resonances.

The chemical structure of PIC, along with its absorption spectra of the monomeric form (red curve) and J-aggregated form (blue curve) are provided in Figure 1. At low concentrations $\left(\mathrm{c} \ll 10^{-5} \mathrm{M}\right.$ ), only the monomer form of PIC is observed, as shown in the red curve. Cyanine dyes, including PIC, have been shown to aggregate in the presence of minerals such as montmillirite clay. ${ }^{36}$ It is presumed that the metal-oxide content in the mineral clays produce a negatively charge surface, and promotes the J-aggregation of cationic cyanine dyes onto the surface of the clay. We employed this approach to induce the J-aggregate formation and mixed a small amount $(100 \mu \mathrm{L})$ of a $0.1 \mathrm{mg} / \mathrm{mL}$ suspension of Halloysite clay in water with $2 \mathrm{~mL}$ of $0.02 \mathrm{mM}$ PIC. A red-shifted J-aggregate peak immediately forms near $575 \mathrm{~nm}$, upon addition of the Halloysite clay, as observed in the blue curve.

In order to explore the interaction between PIC and plasmonic nanoparticles in our study, aliquots of PIC were mixed with solutions containing silver nanoplatelets. Silver nanoplatelets were first fabricated using a common approach in which silver cations are reduced with sodium borohydride in the presence of polyvinylpyrrolidone (PVP), sodium citrate,

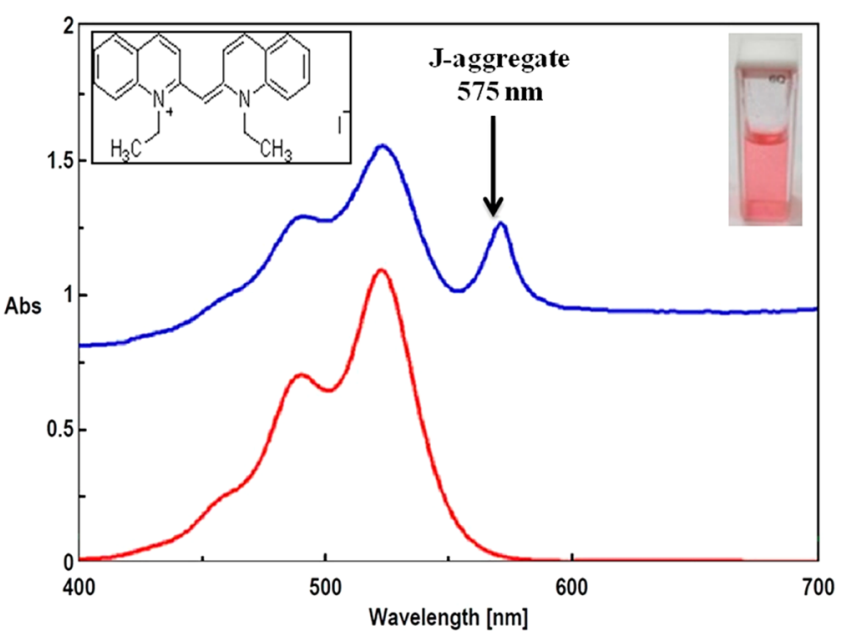

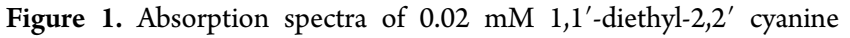
iodide (PIC) in phosphate buffer, $\mathrm{pH}=6$ (red curve). Absorption of the monomer is observed. In the presence of halloysite clay $(1 \mathrm{mg} /$ $\mathrm{mL}$ ), the formation of a J-aggregate is induced and a new, red-shifted peak is observed at $575 \mathrm{~nm}$ (blue curve). Insets provide the chemical structure of PIC (upper left) and an image of the PIC solution within the measurement cuvette (upper right). The optical path length for all measurements was $10 \mathrm{~mm}$.

and hydrogen peroxide. ${ }^{20}$ The PVP and citrate molecules have been shown to adhere to specific crystal faces of the silver as it nucleates and grows, thereby inducing nonspherical nanoparticle geometries. $^{20}$ In addition to the in-house platelet synthesis efforts, silver nanoplatelets were also obtained from Nanocomposix, Inc. (San Diego, CA). A transmission electron microscopy image of silver nanoplatelets synthesized in-house is provided in Figure 2a. Figure $2 \mathrm{~b}$ provides a schematic of the silver platelet core-J-aggregate shell nanostructure. In Figure $2 \mathrm{c}$, the absorption spectrum of a $0.1 \mathrm{mg} / \mathrm{mL}$ silver nanoplatelet solution (Nanocomposix Inc.) is provided in the blue curve, while the red curve represents a mixture containing $2 \mathrm{~mL}$ of a $0.1 \mathrm{mg} / \mathrm{mL}$ silver nanoplatelet solution with $100 \mu \mathrm{L}$ of $0.5 \mathrm{mM}$ PIC. An attenuation dip at $588 \mathrm{~nm}$ emerges when the silver platelets are in the presence of PIC, representing a transparency of $>50 \%$ (peak to dip). The presence of a transparency dip, a blue-shifted high energy peak (relative to the transparency dip), and a red-shifted low energy peak are all characteristic spectral features of plexcitonic structures that exhibit strong plasmonexciton coupling. ${ }^{11,12}$ The transparency and red-shifted lower energy peak are readily observable for the Ag platelet/PIC spectra shown in Figure 2c. Further inspection of the red curve in Figure $2 \mathrm{c}$ reveals the presence of an inflection point at 568 $\mathrm{nm}$, which was hypothesized to be due to the underlying blueshifted plexcitonic peak that was obscured by the presence of the monomer form of the dye. To reveal more clearly the spectral effects of the J-aggregate, we subtracted out the PIC monomer absorption through the standard least-squares background subtraction technique. ${ }^{37}$ The background subtraction technique enabled, for example, the conversion of the red curve in Figure 2c to the " $582 \mathrm{~nm}$ " curve of Figure 3; in the latter, the noisy monomer data is removed, the plexcitonic upper branch is clearly visible, and computation of the peak-todip ratio is straightforward.

In order to explore the impact of the underlying plasmon resonance energy on the degree of plasmon-exciton coupling in our silver platelet/PIC system, PIC was individually mixed with 


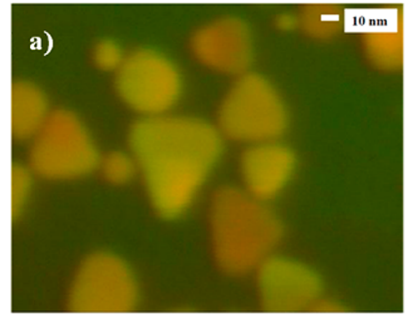

b)

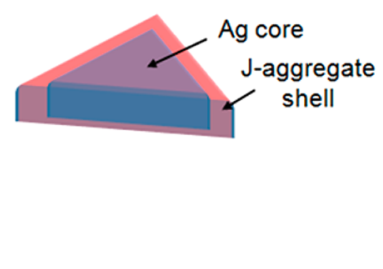

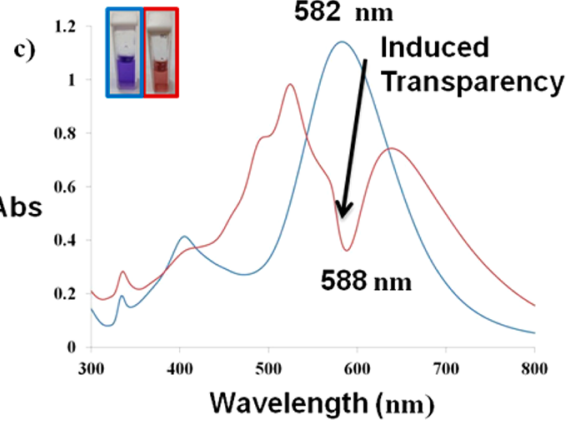

Figure 2. (a) TEM image of silver nanoplatelets (scale bar $=10 \mathrm{~nm}$ ). (b) Schematic of a bilayered nanoplatelet, consisting of a silver nanoplatelet core and a PIC J-aggregate shell. (c) Absorption spectra of a solution containing $0.1 \mathrm{mg} / \mathrm{mL}$ silver nanoplatelets in water (blue curve) and a solution containing $2 \mathrm{~mL}$ of $0.1 \mathrm{mg} / \mathrm{mL}$ silver nanoplatelet solution mixed with $100 \mu \mathrm{L}$ of $0.02 \mathrm{mM}$ PIC (red curve). An induced transparency is observed. The inset (upper left) provides images of the silver nanoplatelet and silver nanoplatelet/PIC solutions. The optical path length for all spectral measurements was $10 \mathrm{~mm}$.

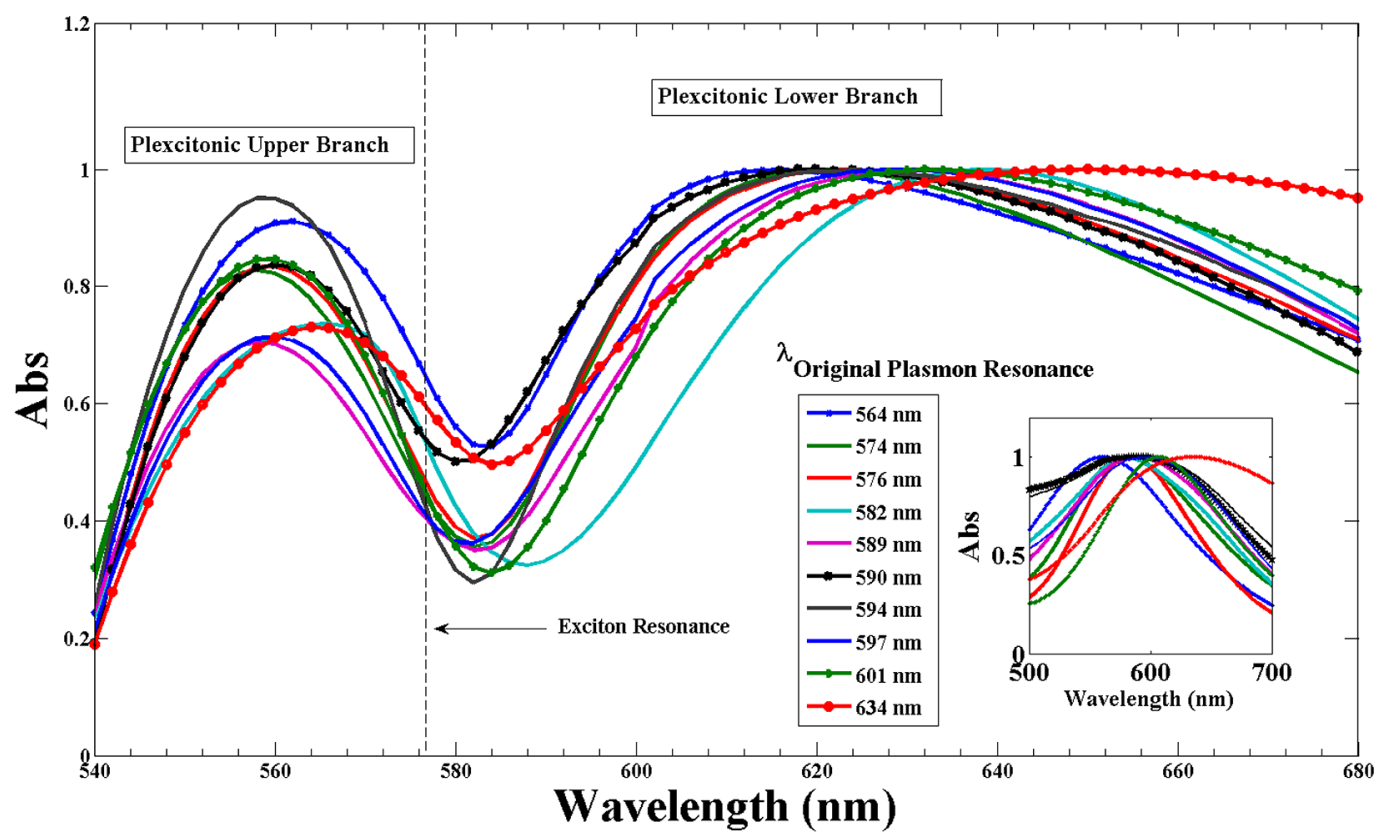

Figure 3. Absorption spectra of silver nanoplatelet/PIC solutions. Each curve represents spectra of $\mathrm{Ag}$ platelet/PIC solutions in which $2 \mathrm{~mL}$ of a given silver nanoplatelet solution were mixed with $60 \mu \mathrm{L}$ of $0.5 \mathrm{mM}$ PIC. By tuning the aspect ratio of the particles, the original resonant plasmonic wavelength (before adding PIC) was varied from $\lambda_{\text {orig }}=564 \mathrm{~nm}$ to $\lambda_{\text {orig }}=634 \mathrm{~nm}$ (as indicated in the legend with original spectra provided in the inset). Adding PIC creates two new peaks, a blue-shifted "upper branch" and a red-shifted "lower branch," that are approximately centered around the resonant wavelength $(\lambda=575 \mathrm{~nm})$ of the J-aggregate.

silver nanoplatelets that varied in aspect ratio. The variation in platelet aspect ratio allowed for the plasmon resonance to be tuned from blue to red, crossing the J-aggregate exciton resonance. Platelet size was controlled by varying the amounts of hydrogen peroxide added during the silver platelet synthetic approach, as described in the Experimental Details. Upon addition of the PIC to each platelet batch, a transparency dip, a blue-shifted higher energy peak, and a red-shifted lower energy peak were consistently generated, as shown in Figure 3. Each curve in Figure 3 represents a different original plasmon resonance, within a specific silver platelet batch.

In general terms, the formation of both a blue-shifted and a red-shifted resonance may be described as the coherent coupling of the plasmon resonance of the silver platelet with the exciton transition dipole of the J-aggregate hybrid. This coupling produces the formation of two plexcitonic modes, an upper branch (UB) mode (blue-shifted peak) and a lower branch (LB) mode (red-shifted peak). ${ }^{38}$ When the plasmon and exciton resonance energies are equal, the energy difference between the upper and lower branches is known as the Rabi splitting energy, $\hbar \omega_{R}$, or as the coupling energy. Two overlapping resonances, and the subsequent formation of two new modes or branches, are often visualized in a fashion that is analogous to how molecular orbital energy diagrams are depicted. ${ }^{38}$ Hence, a hybridized energy diagram depicting the overlap of the plasmon and exciton resonances in our system is provided in Figure 4a. In order to calculate the Rabi splitting energy for the plexcitonic system, the peak resonances of the upper and lower branches were extracted from the experimental curves provided in Figure 3. This was achieved by plotting the upper branch and lower branch energies as a function of the original plasmon resonance peak energy, yielding an upper branch curve and a lower branch curve, as shown in Figure $4 \mathrm{~b}$. Parameters from these curves were then used to calculate the Rabi splitting energy as the difference in upper and lower branch energies for the energy at which the plasmon resonance 

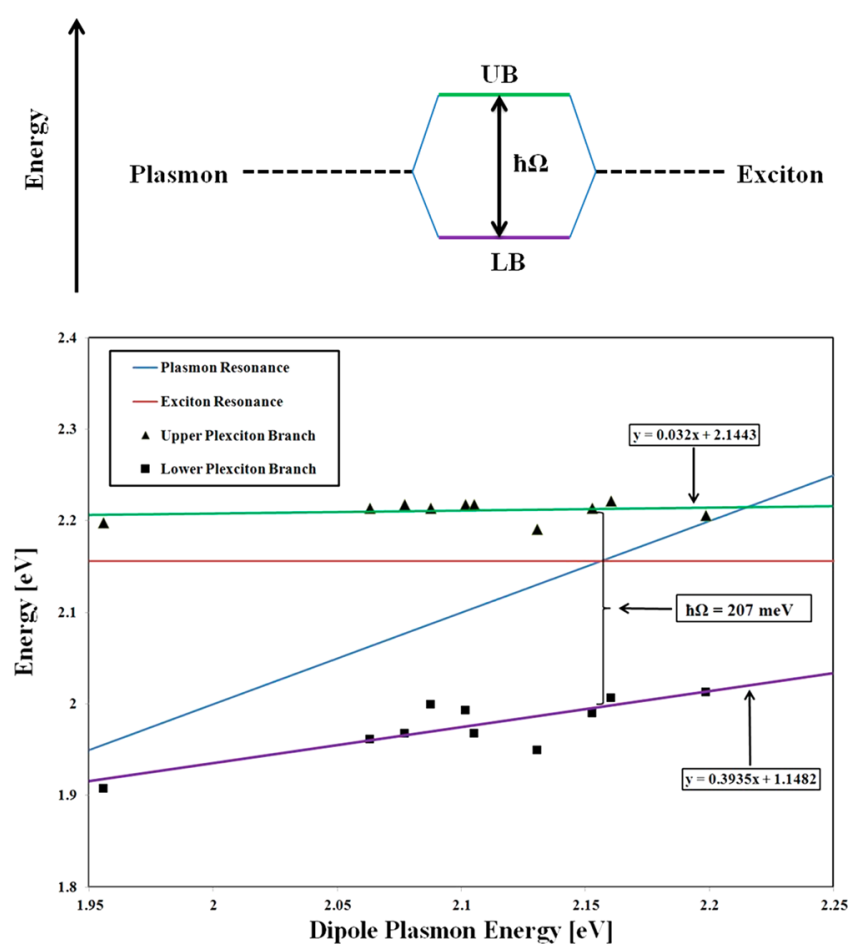

Figure 4. (a) Energy diagram of a plexcitonic system in which the plasmon and exciton resonances overlap. The coupling of these resonances yields upper and lower plexcitonic branches. (b) Calculation of the Rabi splitting energy. Upper and lower branch data points, extracted from Figure 3, are depicted as triangles and squares, respectively. The green and purple curves represent linear fits to these experimentally determined points, respectively. Parameters from these curves were then used to calculate the Rabi splitting energy as the difference between the upper and lower branch energies for the energy at which the plasmon resonance and the exciton resonance were equal, that is, at $575 \mathrm{~nm}$ or $2.156 \mathrm{eV}$. The red and blue curves correspond to the uncoupled exciton and surface plasmon energies, respectively.

and the exciton resonance were equal, that is, at $575 \mathrm{~nm}$ or $2.156 \mathrm{eV}$. A Rabi splitting energy of $207 \mathrm{meV}$ was determined, which is among the highest reported for plexcitonic colloids. ${ }^{12}$

In order to elucidate the optical response of the $\mathrm{Ag} / \mathrm{PIC}$ nanocomposites, numerical and analytical calculations were performed using the boundary-element method and temporal coupled-mode theory, respectively. Both the numerical and analytical simulations predicted the presence of an induced transparency for multilayered nanoplatelets with the morphology and dimensions of those described in the Experimental Details.

For the numerical computations, a free-software implementation $^{39}$ of the boundary element method (BEM) ${ }^{40}$ was employed. BEM calculations revealed that the aspect ratio of the platelet (diameter/thickness) was the primary factor in determining resonant frequencies and cross sections, that is, circular and triangular platelets with equal aspect ratios yielded similar resonant frequencies and cross sections. Hence, the platelets in this study were modeled as cylindrical platelets. The distribution of experimental sizes was modeled by fixing the thickness of the platelets at $10 \mathrm{~nm}$ and varying the diameters of the platelets from 30 to $50 \mathrm{~nm}$. A diameter range of 30-50 nm was chosen for its consistency with TEM images. The TEM images obtained in this study did not sufficiently provide a means of measuring platelet thickness. However, a platelet thickness of $10 \mathrm{~nm}$, determined in a previous study using an identical silver platelet synthesis, was assumed for the computations. ${ }^{20}$ The size-averaged extinction per unit volume is given by adding the extinction per volume ratios of the individual sizes, weighted by the relative volume fraction (taken to be equal). The complex frequency-dependent dielectric function of the PIC J-aggregate was approximated using ${ }^{2,11}$

$$
\varepsilon(\omega)=\varepsilon_{0}+\frac{f \omega_{0}^{2}}{\omega_{0}^{2}-\omega^{2}-i \gamma \omega}
$$

Here, $575 \mathrm{~nm}$ was taken as the excitation wavelength, $\omega_{0}$. TEM images of the PIC-coated silver platelets did not reveal a definitive dye layer thickness. Therefore, a PIC coating thickness of $8 \mathrm{~nm}$, an oscillator strength of $f=0.02$ and a line width $\hbar \gamma=21 \mathrm{meV}$ were chosen as fits to the experimental data. These values were consistent with those used in previous studies. ${ }^{2,11}$ An induced transparency is clearly visible in the computed volume extinction coefficient, as shown in Figure 5.

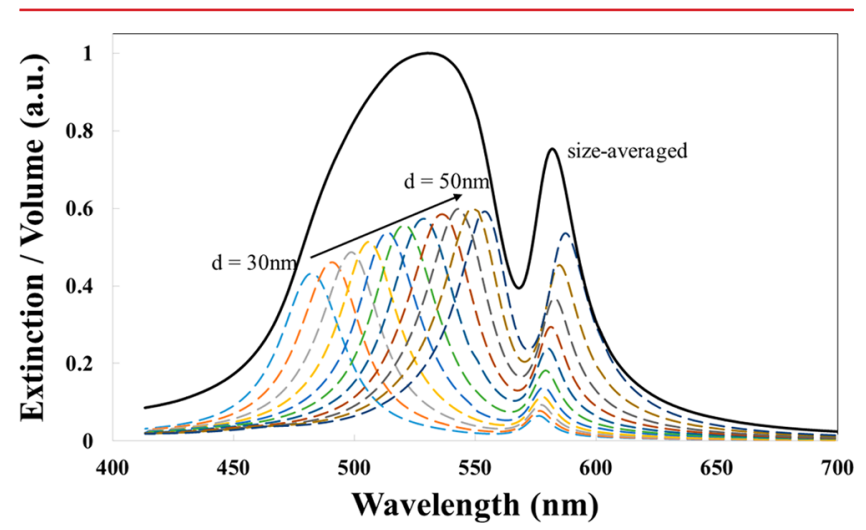

Figure 5. Average extinction cross-section per unit volume of a distribution of PIC-coated silver platelets, computed using the boundary element method. The distribution of experimental sizes was modeled by fixing the thickness of the platelets at $10 \mathrm{~nm}$ and varying the diameter $d$ from 30 to $50 \mathrm{~nm}$. A thickness of $8 \mathrm{~nm}$ was assumed for the PIC coating. The PIC permittivity is modeled by the complex frequency-dependent dielectric function described in the text with an oscillator strength of $f=0.02$ and a line width $\hbar \gamma=21 \mathrm{meV}$. The single-particle data (dashed lines, scaled up by a factor of 3 ) shows significant transparency dips when the exciton and plasmon resonances are close. In the average extinction over an equal distribution of the sizes (solid line), the transparency dip persists due to the relatively small variation in the transparency energy.

The primary limitation to the extent of the transparency dip is the distribution of sizes present in each sample: given a smaller distribution of platelet sizes, an even greater peak to valley ratio is possible. The uniform variation in platelet size used to generate the curves for Figure 5 was merely chosen as a means of explaining the basic features of the experimental spectra, that is, why the resonance is broad and why the transparency dip is persistent across experimental variation. The computations provided in Figure 5 are not intended as a precise model of every aspect of experimental variation, which includes platelet diameter, platelet thickness, coating thickness, coating uniformity, and the distribution of each parameter. None of these parameters were precisely controlled in the experiment, nor could all of these parameters be easily measured. However, our model may easily be generalized, for example, to Gaussian distributions, which would show very similar spectral features to those obtained in Figure 5. 
The suppressed extinction can also be modeled analytically. Since the core-shell structure studied here is much smaller than the wavelength, the absorption and scattering cross sections of the particle are well described by the electric dipole contribution, as

$$
\begin{aligned}
& \sigma_{\mathrm{abs}}=\frac{3 \lambda^{2}}{8 \pi}\left(1-|R|^{2}\right) \\
& \sigma_{\mathrm{sca}}=\frac{3 \lambda^{2}}{8 \pi}|1-R|^{2}
\end{aligned}
$$

When loss is small, the reflection coefficient $R$ can be described using temporal coupled-mode theory as ${ }^{34}$

$$
R=1-2 \frac{\left[i\left(\omega_{2}-\omega\right)+\xi_{2}\right] \gamma_{1}+\left[i\left(\omega_{1}-\omega\right)+\xi_{1}\right] \gamma_{2}}{\left[i\left(\omega_{1}-\omega\right)+\gamma_{1}+\xi_{1}\right]\left[i\left(\omega_{2}-\omega\right)+\gamma_{2}+\xi_{2}\right]-\gamma_{1} \gamma_{2}}
$$

where $\omega$ is the frequency of the incident light, $\omega_{1,2}$ are the resonant frequencies, $\gamma_{1,2}$ are the radiative decay rates, and $\xi_{1,2}$ are the absorptive decay rates. Figure 6 provides a comparison

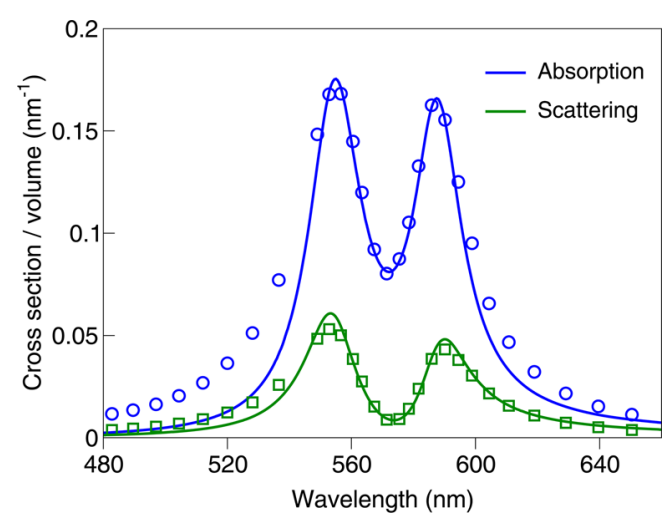

Figure 6. Comparison between temporal coupled-mode theory and BEM. Symbols are calculated using BEM for a platelet with core thickness of $10 \mathrm{~nm}$, core diameter of $50 \mathrm{~nm}$, and a $8 \mathrm{~nm}$ thick layer of PIC coating. Lines are modeled by temporal coupled-mode theory using $\omega_{1}=2.24 \mathrm{eV}, \gamma_{1}=10 \mathrm{meV}, \xi_{1}=31 \mathrm{meV}$ for the plasmonic resonance and $\omega_{2}=2.11 \mathrm{eV}, \gamma_{2}=6.3 \mathrm{meV}, \xi_{2}=27 \mathrm{meV}$ for the exciton resonance.

of the temporal coupled-mode theory results with the BEM calculations. The symbols in Figure 6 represent the single particle cross-section as calculated with BEM using a platelet with a core thickness of $10 \mathrm{~nm}$, a core diameter of $40 \mathrm{~nm}$, and an $8 \mathrm{~nm}$ thick layer of PIC coating. The lines in Figure 6 represent the single particle cross-section as modeled by temporal coupled-mode theory, where the specific parameters $\left(\omega_{1}=2.24 \mathrm{eV}, \gamma_{1}=10 \mathrm{meV}, \xi_{1}=31 \mathrm{meV}\right.$ for the plasmonic resonance, and $\omega_{2}=2.11 \mathrm{eV}, \gamma_{2}=6.3 \mathrm{meV}, \xi_{2}=27 \mathrm{meV}$ for the exciton resonance) are determined by the resonance locations, widths, and heights in the BEM data. Figure 6 shows strong agreement between temporal coupled-mode theory and BEM calculations, and an induced transparency is predicted.

Conclusion. We have reported strong plexcitonic coupling between the localized surface plasmon resonance of silver nanoplatelets and J-aggregate excitons. This coupling produced an induced transparency and yielded a Rabi splitting energy that is among the highest reported for colloidal suspensions. The presence of the transparency, or suppressed extinction, was also predicted by numerical and analytical calculations. These calculations further indicate that the suppressed extinction that is observed in this study are largely dominated by the coupling of absorptive resonances and not the coupling of scattering resonances. Hence, we conclude that the suppressed extinction in this study should not be termed a "Fano resonance." The large Rabi energy is explained at least in part by our use of small (almost quasistatic) nanoplatelets, which have nearly optimal absorption response, and for the same resonant frequency yield greater field intensities than coated spheres. ${ }^{41}$ We hypothesize that the strong coupling may also be due in part to the orientation of the J-aggregate along the major dimensions of the platelets, that is, the transition dipoles of the exciton resonance if aligned with the plasmon resonance of the platelet produces an enhanced plexcitonic coupling. Future efforts in modeling the dye orientation on the surface silver platelets should provide insight into the impact of dye orientation on plexcitonic coupling.

Experimental Details. Sample Preparation. (1) PIC standard and PIC/Halloysite clay mixture. A $0.5 \mathrm{mM}$ PIC stock solution was prepared by dissolving $23 \mathrm{mg}$ of $1,1^{\prime}$-diethyl2,2'-cyanine iodide (Sigma-Aldrich) in $100 \mathrm{~mL}$ phosphate buffer $(\mathrm{pH}=6.0)$. A $0.01 \mathrm{mM}$ PIC standard solution was prepared by diluting $1 \mathrm{~mL}$ of the $0.5 \mathrm{mM}$ PIC stock solution with phosphate buffer in a $50 \mathrm{~mL}$ volumetric flask. In order to induce the formation of the J-aggregate form of PIC, $2 \mathrm{~mL}$ of the $0.01 \mathrm{mM}$ PIC standard solution was mixed with $100 \mu \mathrm{L}$ of a $1 \mathrm{mg} / \mathrm{mL}$ suspension containing Halloysite clay (SigmaAldrich) in water. (2) Silver nanoplatelet/PIC mixtures. Two milliliters of a $0.1 \mathrm{mg} / \mathrm{mL}$ silver nanoplatelet solution (Nanocomposix Inc.) was mixed with $100 \mu \mathrm{L}$ of $0.5 \mathrm{mM}$ PIC, yielding the spectra provided in Figure 2c. (3) Silver nanoplatelets were also synthesized in-house using a modified version of Mirkin's approach. ${ }^{20}$ Briefly, $25 \mathrm{~mL}$ of a $0.11 \mathrm{mM}$ $\mathrm{AgNO}_{3}$ (Sigma-Aldrich) was placed in a $2 \mathrm{oz}$. Wheaton jar. While magnetically stirring at room temperature, $1.5 \mathrm{~mL}$ of a 30 $\mathrm{mM}$ sodium citrate (Sigma-Aldrich) solution in water, $1.5 \mathrm{~mL}$ of a $10 \mathrm{mg} / \mathrm{mL}$ polyvinylpyrollidone (MW $=29000$, Aldrich) solution in water, and a variable amount (ranging from 20 to 40 $\mu \mathrm{L})$ of $30 \%(\mathrm{w} / \mathrm{w})$ hydrogen peroxide (Sigma-Aldrich) were added. The mixtures were stirred for $15 \mathrm{~min}$, after which 100 $\mu \mathrm{L}$ of $100 \mathrm{mM} \mathrm{NaBH}_{4}$ (Sigma-Aldrich) was added to each solution. The solutions were stirred for $24 \mathrm{~h}$. The variable amount of hydrogen peroxide produced silver platelets with varying size, as measured by dynamic light scattering. Specifically, the addition of the variable amount of $30 \%$ hydrogen peroxide solution yielded average platelet sizes from 34.5 to $58.2 \mathrm{~nm}$. (4) Synthesized silver nanoplatelets/PIC mixtures. Two milliliters of the final silver platelet solutions $(\sim 0.01 \mathrm{mg} / \mathrm{mL})$ were mixed with $60 \mu \mathrm{L}$ of $0.5 \mathrm{mM}$ PIC.

\section{AUTHOR INFORMATION}

\section{Corresponding Author}

*E-mail: brendan.g.delacy.civ@mail.mil.

\section{Funding}

This research was funded by the Department of the Army Basic Research Program and sponsored by the Edgewood Chemical Biological Center. Support was also provided by the U.S. Army Research Office through the Institute for Soldier Nanotechnologies under Contract No. W911NF-13-D-0001.

\section{Notes}

The authors declare no competing financial interest. 


\section{REFERENCES}

(1) Kometani, N.; Tsubonishi, M.; Fujita, T.; Asami, K.; Yonezawa, Y. Langmuir 2001, 17, 578-580.

(2) Widerrecht, G. P.; Wurtz, G. A.; Hranisavljevic, J. Nano Lett. 2004, 4, 2121-2125.

(3) Yoshida, A.; Kometani, N.; Yonezawa, Y. Colloids Surf., A 2008, 313-314, 581-584.

(4) Lebedev, W. S.; Vitukhnovsky, A. G.; Yoshida, A.; Kometani, N.; Yonezawa, Y. Colloids Surf., A 2008, 326, 204-209.

(5) Yoshida, A.; Yonezawa, Y.; Kometani, N. Langmuir 2009, 25, 6683-6689.

(6) Yoshida, A.; Uchida, N.; Kometani, N. Langmuir 2009, 25, 11802-11807.

(7) Achermann, M. J. Phys. Chem. Lett. 2010, 1, 2837-2843.

(8) DeLacy, B. G.; Qiu, W.; Soljacic, M.; Hsu, C. W.; Miller, O. W.; Johnson, S. G.; Joannopoulos, J. D. Opt. Express 2013, 21, 1910319112.

(9) Lu, Z.; Zhu, K. J. Phys. B: At. Mol. Opt. Phys. 2008, 41, 185503.

(10) Kohlgraf-Owens, D. C.; Kik, P. G. Opt. Express 2008, 16, 10823-10834.

(11) Fofang, N. T.; Grady, N. K.; Fan, Z.; Govorov, A. O.; Halas, N. J. Nano Lett. 2011, 11, 1556-1560.

(12) Zengin, G.; Johansson, G.; Johansson, P.; Antosiewics, T. J.; Kall, M.; Shegai, T. Sci. Rep. 2013, 3 (3074), 1-8.

(13) Murphy, C. J. Anal. Chem. 2002, 74, 520 A-526A.

(14) Govorov, A. O.; Carmeli, I. Nano Lett. 2007, 7, 620-625.

(15) Slocik, J. M.; Tam, F.; Halas, N. J.; Naik, R. R. Nano Lett. 2007, 7, 1054-1058.

(16) Artuso, R. D.; Bryant, G. W. Nano Lett. 2008, 8, 2106-2111.

(17) Orendorff, C. J.; Gearheart, L.; Jana, N. R.; Murphy, C. J. Phys. Chem. Chem. Phys. 2006, 8, 165-170.

(18) Au, L.; Chen, Y.; Zhou, F.; Camargo, Pl H. C.; Lim, B.; Li, Z.; Ginger, D. S.; Xia, Y. Nano Res. 2008, 1, 441-449.

(19) Khoury, C. G.; Vo-Dinh, T. J. Phys. Chem. C 2008, 112, 1884918859.

(20) Metraux, G. S.; Mirkin, C. A. Adv. Mater. 2005, 17, 412-415.

(21) Davydov, A. S. Theory of Molecular Excitons; Plenum Press: New York, 1971.

(22) Van Burgel, M.; Wiersma, D. A.; Duppen, K. J. Chem. Phys. 1995, 102, 20-33.

(23) Artar, A.; Yanik, A. A.; Altug, H. Nano Lett. 2011, 11, 16851689.

(24) Tiannini, V.; Francescato, Y.; Amrania, H.; Phillips, C. C.; Maier, S. A. Nano Lett. 2011, 11, 2835-2840.

(25) Gallinet, B.; Martin, O. J. F. Phys. Rev. B 2011, 83, 235427.

(26) Gallinet, B.; Martin, O. J. F. ACS Nano 2011, 5, 8999-9008.

(27) Davis, T. J.; Gomez, D. E.; Vernon, K. C. Nano Lett. 2010, 10, 2618-2625.

(28) Forestiere, C.; Dal Negro, L.; Miano, G. Phys. Rev. B 2013, 88, 155411.

(29) Mukherjee, S.; Sobhani, H.; Lassiter, J. B.; Bardhan, R.; Nordlander, P.; Halas, N. J. Nano Lett. 2010, 10, 2694-2701.

(30) Artar, A.; Yanik, A. A.; Altug, H. Nano Lett. 2011, 11, 36943700 .

(31) Lassiter, J. B.; Sobhani, H.; Knight, M. W.; Mielczarek, W. S.; Nordlander, P.; Halas, N. J. Nano Lett. 2012, 12, 1058-1062.

(32) Lovera, A.; Gallinet, B.; Nordlander, P.; Martin, O. J. ACS Nano 2013, 7, 4527-4536.

(33) Adato, R.; Artar, A.; Erramili, S.; Altug, H. Nano Lett. 2013, 13, 2584-2591.

(34) Fan, S.; Suh, W.; Joannopoulos, J. D. J. Opt. Soc. Am. A 2003, 20, 569-572.

(35) Hsu, C. W.; DeLacy, B. G.; Johnson, S. G.; Joannopoulos, J. D.; Soljacic, M. Nano Lett. 2014, 14, 2783-2788.

(36) Dixon, A.; Duncan, C.; Samha, H. Am. J. Undergr. Res. 2005, 3, 29-34.

(37) Statham, P. J. Anal. Chem. 1977, 49, 2149-2154.

(38) Schlather, A. E.; Large, N.; Urban, A. S.; Nordlander, P.; Halas, N. J. Nano Lett. 2013, 13, 3281-3286.
(39) Reid, M. T. H. http://homerreid.com/scuff-EM (accessed Nov $6,2014)$.

(40) Harrington, R. F. Field Computations by Moment Methods; IEEE Press: Piscataway, NJ, 1993.

(41) Miller, O. D.; Hsu, C. W.; Reid, M. T. H.; Qiu, W.; DeLacy, B. G.; Joannopoulos, J. D.; Soljacic, M.; Johnson, S. G. Phys. Rev. Lett. 2014, 112, 123903.

(42) Balci, S. Opt. Lett. 2013, 38, 4498-4501.

\section{NOTE ADDED IN PROOF}

After this manuscript was accepted, it came to our attention that a related study was recently published. We have included this work as an additional reference. ${ }^{42}$ 